\title{
PERIDYNAMIC MODELLING OF FRACTURE IN POLYCRYSTALLINE ICE
}

Wei Lu${ }^{1,2}$, Mingyang $\mathrm{Li}^{1}$, Bozo Vazic ${ }^{1}$, Selda Oterkus ${ }^{1}$, Erkan Oterkus ${ }^{1}$ and Qing Wang ${ }^{2}$

${ }^{1}$ University of Strathclyde, Glasgow, UK

${ }^{2}$ College of shipbuilding Engineering, Harbin Engineering University, Harbin, China

\begin{abstract}
In this study, a peridynamic material model for a polycrystalline ice is utilised to investigate its fracture behaviour under dynamic loading condition. First, the material model was validated by considering a single grain, double grains and polycrystalline structure under tension loading condition. Peridynamic results are compared against finite element analysis results without allowing failure. After validating the material model, dynamic analysis of a polycrystalline ice material with two pre-existing cracks under tension loading is performed by considering weak and strong grain boundaries with respect to grain interiors. Numerical results show that the effect of microstructure is significant for weak grain boundaries. On the other hand, for strong grain boundaries, the effect of microstructure is insignificant. The evaluated results have demonstrated that peridynamics can be a very good alternative numerical tool for fracture analysis of polycrystalline ice material.
\end{abstract}

Keywords: Peridynamics; Polycrystalline ice; Fracture; Numerical. 


\section{INTRODUCTION}

In Arctic regions, the accurate prediction of ice behaviour plays a significant role in the optimum design of marine structures and polar ships. The naturally generated ice has usually polycrystalline structure which can be characterized by its average grain size, grain morphology, and grain orientation, and is highly dependent on the properties of grain boundary which can be associated with microcracks [1-3]. Therefore, it is a great challenge to fully understand the behaviour of polycrystalline ice.

In order to study the behaviour of polycrystalline material, various experiments have been done by using Xray analysis [4-5]. However, although the results obtained from experiments provide very useful information for the future studies, the expensive cost of equipment and time-consuming procedures of preparation of the material limit its wide application. Currently, with the development of high-performance computing, the numerical simulations become one of the predominant approaches utilized in the polycrystalline material analysis. Amongst the computational techniques, the cohesive zone model (CZM) within the framework of the finite element method (FEM) is one of the most widely used approaches for fracture analysis of polycrystalline systems. A twodimensional finite element model was developed by Warner and Molinari [6] to investigate intergranular fracture in alumina with the grain interiors modelled as anisotropic elastic material and the grain boundary properties were fitted with experimental data. Sfantos and Aliabadi [7] proposed boundary cohesive grain element model to simulate the intergranular fracture of $\mathrm{SiC}$. To investigate the transition from an intergranular to a transgranular mode of fracture, the extended finite element method (XFEM) was used to model quasi-static crack propagation [8]. While to study the fracture of ice, in addition to XFEM and CZM, the discrete element method (DEM) and smoothed particle hydrodynamics method (SPH) are used in numerical simulations as well, especially for the icestructure interaction. The analysis of ice sheet failure and ice rubble pile formation progress against the inclined structure was done by Paavilainen et al. [9-10] in the two dimensional numerical simulation based on the discrete element method. Lu et al. [11] and Feng et al. [12] applied a three dimensional finite element model with the use of cohesive element method to develop the numerical model for level ice and offshore structures. SPH is utilized in the numerical simulation of the bending test of ice beam [13]. For the polycrystalline ice, Gribanov et al. [14] implemented the cohesive zone model within an implicit finite element method to simulate the 4-point bending test of freshwater ice beam. Furthermore, the same CZM model is utilized by Gribanov et al. [15] to model the fracture behaviour of polycrystalline cylindrical samples under uniaxial loading conditions. The numerical results capture the observations fracture from the experiment. Although all these approaches provide useful information, since they are based on classical continuum mechanics, they inherit certain disadvantages of classical continuum mechanics [16]. Specifically, the governing equations of the classical continuum mechanics incorporate spatial displacement derivatives which are undefined along the crack surfaces. Moreover, CZM has mesh dependency issues. Although this problem can be overcome by using XFEM, its application for 3-Dimensional models is challenging. The XFEM is based on the generalized finite element method. It adds a local enrichment function with discontinuous displacement to represent the discontinuous area in the domain to be solved. However, for the three dimensional model, the progress of enrichment may result in an algebraic system with a large number of unknown variables which is difficult to solve. By taking these difficulties into consideration, a non-local meshfree method proposed by Silling [17], called peridynamics, can be utilized for the simulation of the polycrystalline ice. In this formulation, integral equations are used instead of the partial differential equations. Therefore, it is suitable for predicting crack initiation and propagation which may occur spontaneously. Askari et al. [18] and De Meo et al. [19] simulated two dimensional polycrystalline fracture in silicon and AISI 4340 steel, respectively, by using peridynamics for cubic crystal systems. Furthermore, Ghajari et al. [20] presented a two dimensional bond-based peridynamic formulation suitable for hexagonal crystal systems.

In this paper, a two dimensional polycrystalline ice model is proposed within the framework of the bond-based peridynamic theory. The peridynamic parameters are obtained by equating the strain energy density of a material point with the classical continuum mechanics. The numerical results of the simulation are compared with the results obtained from FEM to testify the accuracy of the numerical peridynamic ice model. Finally, using the peridynamic polycrystalline ice model, the failure mode of ice under dynamic loading condition is investigated.

\section{PERIDYNAMIC THEORY}

The peridynamic theory was first introduced by Silling [17] using an integral equation as a reformulation of the interactions between material points. A concept of a horizon is introduced in which the material points interact through bonds, whereas the interactions disappear outside the horizon. For bond-based peridynamic theory, the governing equation of a material point at position $\mathbf{x}$ in the reference configuration can be written as

$$
\rho(\mathbf{x}) \ddot{\mathbf{u}}(\mathbf{x}, t)=\int_{H_{\mathbf{x}}} \mathbf{f}\left(\mathbf{u}\left(\mathbf{x}^{\prime}, t\right)-\mathbf{u}(\mathbf{x}, t), \mathbf{x}^{\prime}-\mathbf{x}\right) d V_{\mathbf{x}^{\prime}}+\mathbf{b}(\mathbf{x}, t)
$$


where $\rho$ is the mass density of the reference configuration, $\mathbf{u}$ represents the displacement vector field, $\mathbf{b}$ is the body force, and $\mathbf{f}$ represents the pairwise force function showing the force per volume squared that the particle $\mathbf{x}^{\prime}$ exerts on particle $\mathbf{x}$. $H_{\mathbf{X}}$ is the spherical neighbourhood with a radius of $\delta$ centered at material point $\mathbf{x}$, called horizon. The relative position vector in the original configuration is denoted by $\xi=\mathbf{x}^{\prime}-\mathbf{x}$ and the relative displacement vector at time $t$ is defined as $\boldsymbol{\eta}=\mathbf{u}\left(\mathbf{x}^{\prime}, t\right)-\mathbf{u}(\mathbf{x}, t)$.

The peridynamic forces between two material points interacting with each other are equal in magnitude and in the opposite directions. These forces are along the direction of the relative position vector in the current configuration and can be expressed as

$$
\mathbf{f}(\boldsymbol{\eta}, \xi)=f(\boldsymbol{\eta}, \xi) \frac{\boldsymbol{\eta}+\xi}{\|\boldsymbol{\eta}+\xi\|}
$$

For a microelastic material, the pairwise force function is derivable from the micropotential function $w(\boldsymbol{\eta}, \xi)[17]$ and can be written as

$$
\mathbf{f}(\boldsymbol{\eta}, \xi)=\frac{\partial w}{\partial \boldsymbol{\eta}}(\boldsymbol{\eta}, \xi) \quad \forall \boldsymbol{\eta}, \boldsymbol{\xi}
$$

For a prototype microelastic brittle material proposed by Silling and Askari [21], the pairwise force function is assumed to be dependent on the stretch between material points and can be defined as

$$
\mathbf{f}(\boldsymbol{\eta}, \xi)=c(\xi) s(\boldsymbol{\eta}, \boldsymbol{\xi}) \frac{\boldsymbol{\eta}+\xi}{\|\boldsymbol{\eta}+\xi\|}
$$

in which $s$ is the stretch of the bond which can be expressed as

$$
s(\boldsymbol{\eta}, \xi)=\frac{\|\boldsymbol{\eta}+\xi\|-\|\xi\|}{\|\xi\|}
$$

and $c$ represents the bond constant. By equating the strain energy density of an individual material point from peridynamics with classical continuum mechanics, the bond constant $c$ for a 2 Dimensional isotropic material can be written as

$$
c(\xi)=\frac{6 E}{\pi \delta^{3} b(1-v)}
$$

where $E$ represents the elastic modulus of the material, $b$ is the thickness of the plate, and $v$ is the Poisson's ratio which is limited to $1 / 3$ in the bond-based peridynamic model.

To represent failure of a bond, the critical stretch parameter of the bond $s_{0}$ is introduced. According to Silling and Askari [21], the critical stretch can be related to the critical energy release rate $G_{c}$ as

$$
s_{0}=\sqrt{\frac{4 \pi G_{c}}{9 E \delta}}
$$

For the polycrystalline ice, the failure mode can be different depending on the strength of the grain boundaries. Therefore, a grain boundary coefficient (GBC) is introduced to investigate the fracture of the polycrystalline ice as

$$
\mathrm{GBC}=\frac{s_{0 \mathrm{~GB}}}{s_{0 \mathrm{GI}}}
$$

where $s_{0 \mathrm{~GB}}$ and $s_{\mathrm{OGI}}$ stand for the critical stretch of the peridynamic bonds that cross the grain boundaries and the critical stretch of the peridynamic bonds associated with the grain interior, respectively.

\section{ICE PROPERTIES}

Ice is a complex material consisting of fresh water ice, gas, brine, and different types of solid salts, and shows dependence on the temperature. A single ice crystal behaves as a strong anisotropic material due to its dislocation glide on the basal plane, which is perpendicular to the crystal hexagonal symmetry axis, named as c-axis [22]. During the time of the ice growth, environmentally dominated variations as well as the thermal and deformation history lead to the formation of different grain structures of ice. The most common grain structures include granular, columnar, and discontinuous columnar [23]. In this study, the freshwater columnar grained ice is considered. This type of ice is usually found in the lower layers of lakes and rivers. The ice is composed of columnar crystals which may elongate through the whole thickness of the level ice along the vertical direction. The c-axis of ice crystal are oriented randomly on the plane perpendicular to the direction of the columns. Thus, the columnar ice shows transversely isotropic material behaviour. 


\section{PERIDYNAMIC MICROMECHANICAL MATERIAL MODEL FOR ICE CRYSTALS}

In this study, a peridynamic micromechanical material model is utilised to represent the behaviour of the polycrystalline ice with random crystal orientations. In the polycrystalline structure, each grain is represented using bond-based peridynamics and introducing different bond properties along the crystal orientation direction and other directions. Thus, the peridynamic bonds are divided into two types as shown in Figure 1. The type 1 bonds (shown with orange colour) exist in all the directions and describes the interaction between material points $i$ and all other material points in the horizon denoted by the peridynamic bond constant $c_{1}$. Type 2 bonds (shown with pink colour) only exist along the crystal orientation direction, $\theta$. Therefore, the effect of the crystal orientation can be represented by the bond constant $c_{2}$. The peridynamic constitutive model for the in-plane interactions between two material points can be expressed using Equation (4) by assigning the relevant bond constant value depending on the bond orientation with respect to crystal orientation. Thus, the total bond constant parameter $c$ can be expressed as $[24,30]$,

$$
c= \begin{cases}c_{1}+c_{2} & \text { for } \phi=\theta \\ c_{1} & \text { for } \phi \neq \theta\end{cases}
$$

According to Oterkus and Madenci [24], by equating the strain energy densities of a material point based on classical continuum mechanics with bond-based peridynamics under simple loading conditions, the peridynamic material constants $c_{1}$ and $c_{2}$ can be expressed by the reduced stiffness matrix, $Q_{i j}$ as

$$
\begin{aligned}
& c_{1}=\frac{24 Q_{12}}{\pi b \delta^{3}} \\
& c_{2}=\frac{Q_{11}-Q_{22}}{\beta}
\end{aligned}
$$

where

$$
\beta=\frac{1}{2} \sum_{q=1}^{m} \xi_{q i} V_{q}
$$

in which $m$ represent the number of bonds along the crystal orientation direction within the horizon $\delta$ of material point $i . \xi_{q i}$ is the initial length of the bond along the crystal orientation direction between material point $q$ and $i$, $V_{q}$ donates the volume of the material point $q$, and $b$ is the thickness.

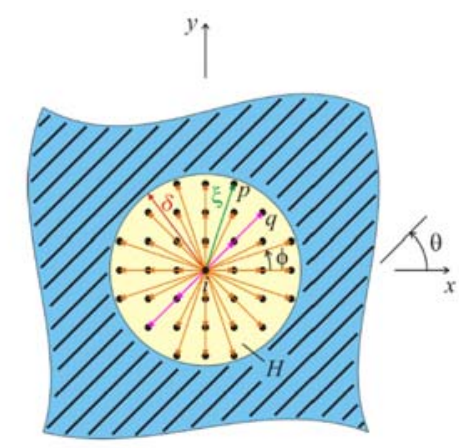

Fig. 1 Horizon of the material point $i$ with a crystal orientation of $\theta$

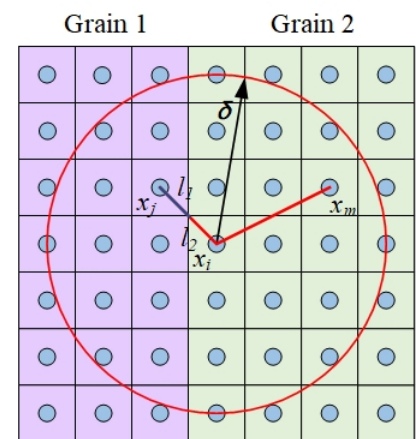

Fig. 2 The sketch of the bond that cross the grain boundary

For the bond that crosses the grain boundary as shown in Figure 2, since the material point $i$ is associated with Grain 2 and material point $j$ is associated with Grain 1, the bond properties differ from the one that are only embedded in a single grain. The bond constant $c_{1}$ is same for the entire plate due to its independence from grain orientation. However, for the bond constant $c_{2}$, it needs to be determined again. According to Oterkus et al. [25] in which the thermal conductivity $k$ of the bond that belongs to two different materials is calculated with the length of the segment of the bond in each material, written as the following equation (Equation 12), the bond constant of the crossing bond which is composed by the particles in two different grains is defined by the similar idea;

$$
k=\frac{l_{1}+l_{2}}{\frac{l_{1}}{k_{1}}+\frac{l_{2}}{k_{2}}}
$$

in which $l_{1}$ represents the segment of the distance between particles $x_{i}$ and $x_{j}$ in material 1 , while $l_{2}$ represents the segment in material 2. $k_{1}$ and $k_{2}$ stand for the thermal conductivity of material 1 and 2 respectively. 
The equivalent bond constant of the crossing bond which is composed by the particles in two different grains can be calculated by using the similar idea. Since each grain of ice is presented as a polygon, it will be computationally very expensive to define length of each bond segments. In order to simplify the model, the length of $l_{l}$ can be assumed to be equal to length of $l_{2}$ and equivalent bond constant, $c_{2 e q}$ can be calculated as

$$
c_{2 e q}=\frac{2 k_{1} k_{2}}{k_{1}+k_{2}}-c_{1}
$$

The parameters $k_{1}$ and $k_{2}$ depend on the crystal orientation of the grains. If the crystal orientation in grain 2 matches with bond orientation between $\mathbf{x}_{i}$ and $\mathbf{x}_{j}$, then

$k_{1}=c_{1}$

$k_{2}=c_{1}+c_{2}\left(\mathbf{x}_{i}\right)$

If the crystal orientation in grain 1 matches with bond orientation between $\mathbf{x}_{i}$ and $\mathbf{x}_{j}$, then

$$
\begin{aligned}
& k_{1}=c_{1}+c_{2}\left(\mathbf{x}_{j}\right) \\
& k_{2}=c_{1}
\end{aligned}
$$

If the crystal orientations in grain 1 and 2 match with bond orientation between $\mathbf{x}_{i}$ and $\mathbf{x}_{j}$, then

$$
\begin{aligned}
& k_{1}=c_{1}+c_{2}\left(\mathbf{x}_{j}\right) \\
& k_{2}=c_{1}+c_{2}\left(\mathbf{x}_{i}\right)
\end{aligned}
$$

If the crystal orientations in grain 1 and 2 does not match with bond orientation between $\mathbf{x}_{i}$ and $\mathbf{x}_{j}$, then

$$
\begin{gathered}
k_{1}=c_{1} \\
k_{2}=c_{1} \\
\text { and } \\
c_{2 e q}=0
\end{gathered}
$$

\section{NUMERICAL RESULTS AND DISCUSSION}

In this section, three static problems are considered by using bond-based peridynamic polycrystalline ice material model described above. The numerical results are compared with the results obtained from FEM in order to verify the accuracy of the peridynamic polycrystalline ice model. For the polycrystalline ice simulations, the polycrystalline structure is generated by implementing the Voronoi tessellation method. The Voronoi diagram is a fundamental geometric structure widely used in the simulation polycrystalline materials, including polycrystalline ice [26-27]. For a given set of seeds in a domain, a Voronoi Tessellation is used to divide the domain into different Voronoi cells. Each of the cells stands for a single crystal, it includes the particles that have shorter distance to the corresponding seed inside the cell than to any other seed.

The reduced stiffness matrix of a single grain can be written as

$$
[Q]=\left[\begin{array}{ccc}
Q_{11} & Q_{12} & 0 \\
Q_{12} & Q_{22} & 0 \\
0 & 0 & Q_{66}
\end{array}\right]
$$

According to Elvin [28], the stiffness properties of a polycrystalline ice can be specified as $Q_{11}=12.624 \mathrm{GPa}$ and $Q_{22}=10.328 \mathrm{GPa}$.

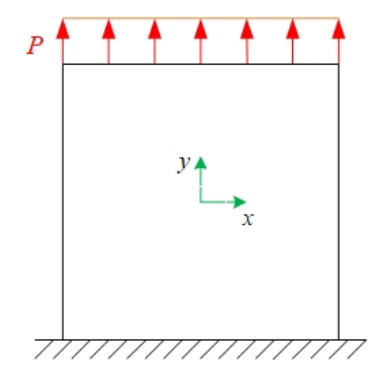

Fig. 3 Single grain for static analysis

\subsection{Single grain under uniaxial tension}


The aim of this example is to show the accuracy of the elastic behaviour of the peridynamic columnar ice model. The crystal employed in this case has a length of $1.2 \mathrm{~mm}$ and a width of $1.2 \mathrm{~mm}$. It is discretized uniformly with 200 particles distributed along the horizontal and vertical directions. Three layers of virtual particles are added along the bottom edge of the plate and set with zero displacements to constrain the bottom edge of the plate. A vertical load of $P=600 \mathrm{MPa}$ is applied as a body force and exerted to the top edge of the plate as the boundary condition as shown in Figure 3. The quasi-static solution is obtained by implementing the adaptive dynamic relaxation method. A constant horizon radius of $\delta=1.809 \times 10^{-5} \mathrm{~m}$ is used corresponding to 3.015 times of the grid spacing as suggested by Madenci and Oterkus [29].

The comparison of the horizontal and vertical displacements between the numerical results obtained from peridynamic and FEM simulations for a single grain with a 45-degree crystal orientation are shown in Figures 4 and 5 .
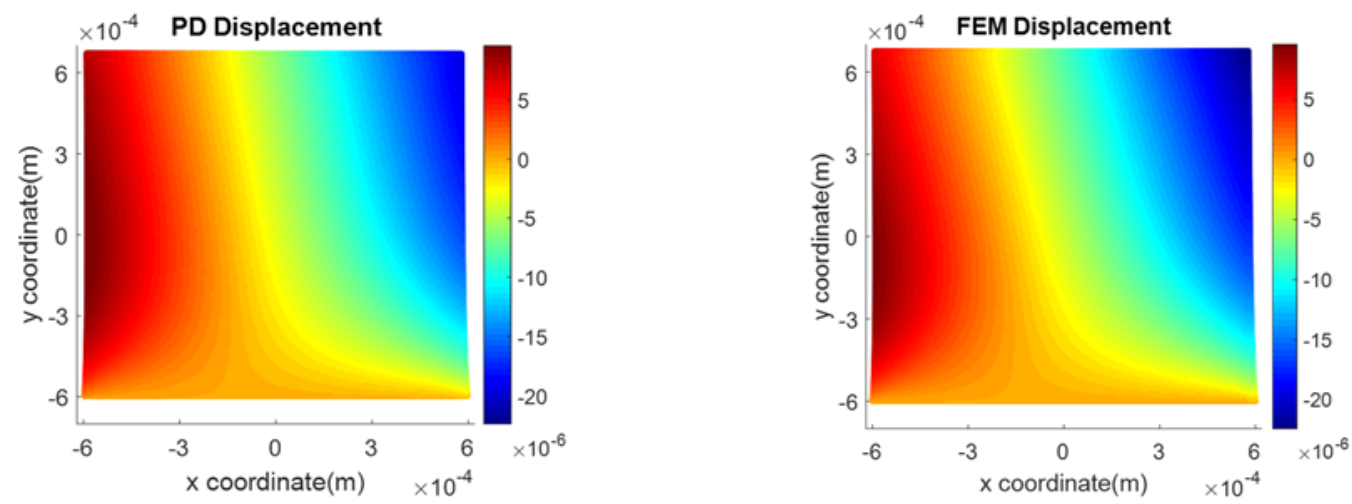

(a) Comparison of horizontal displacement field for a single grain
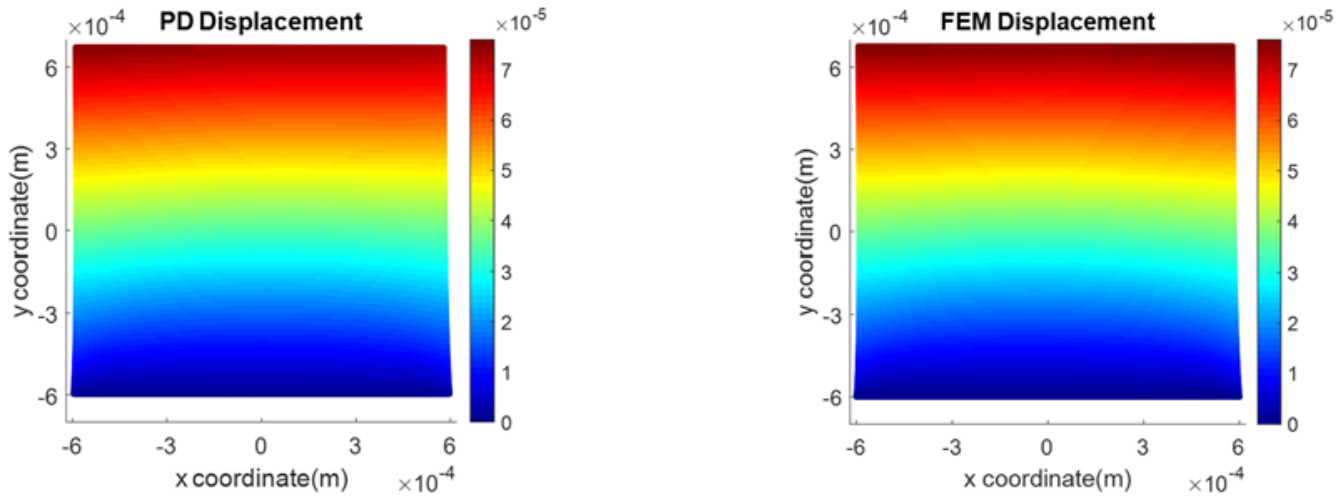

(b) Comparison of vertical displacement field for a single grain

Fig. 4 Comparison of displacement fields between PD and FEM for a single grain
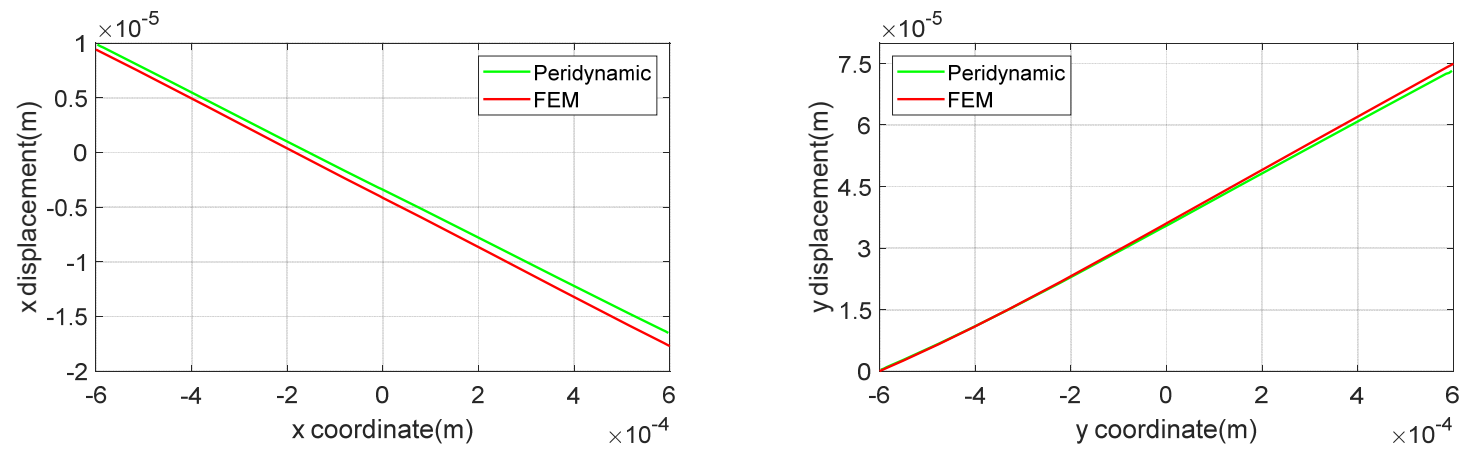

Fig. 5 Comparison of horizontal and vertical displacements along the central axes between PD and FEM for a single grain

It is clear that the numerical results obtained from peridynamic simulation agree well with FEM results for a 
single ice grain under uniaxial tension loading. Therefore, the accuracy of the micromechanical peridynamic model for single grain is successfully verified.

\subsection{Double grain model under uniaxial tension}

In this section, double grain model with crystal orientations of -45 and 45 degrees are considered with a length of $2.4 \mathrm{~mm}$ and a width of $1.2 \mathrm{~mm}$. The number of particles distributed uniformly along the horizontal and vertical directions is 300 and 150, respectively. The bottom edge of the plate is constrained with three layers of virtually added particles. The uniaxial tension loading of $P=600 \mathrm{MPa}$ is applied as body force on the top edge of the plate. The horizon radius is defined as $\delta=2.412 \times 10^{-5} \mathrm{~m}$, i.e. 3.015 times of the grid spacing of $\Delta x=0.008 \mathrm{~mm}$.
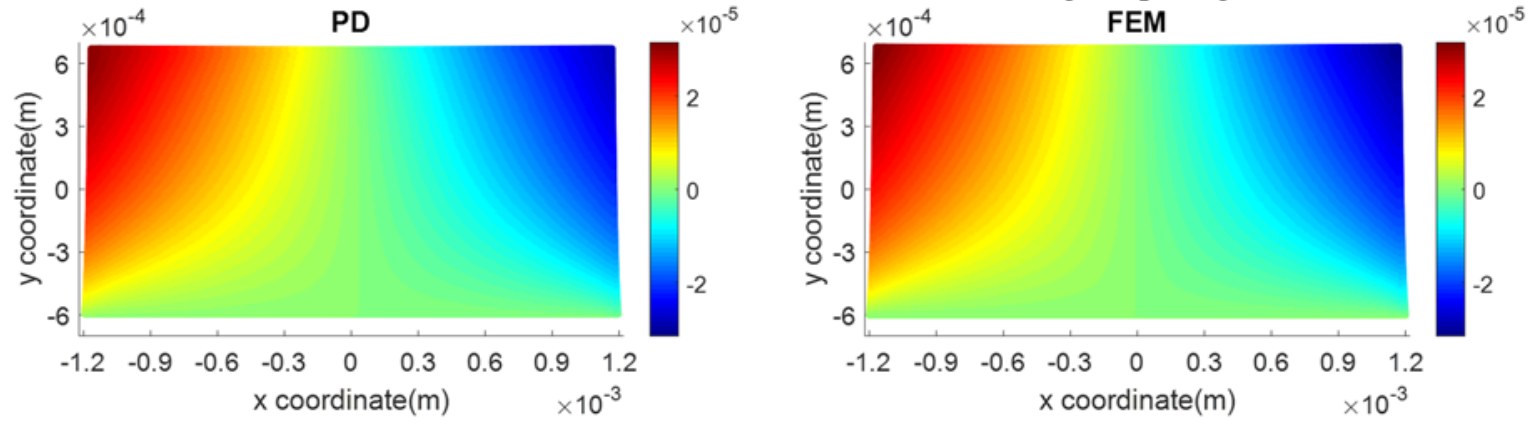

(a) Comparison of horizontal displacement field for double grain model
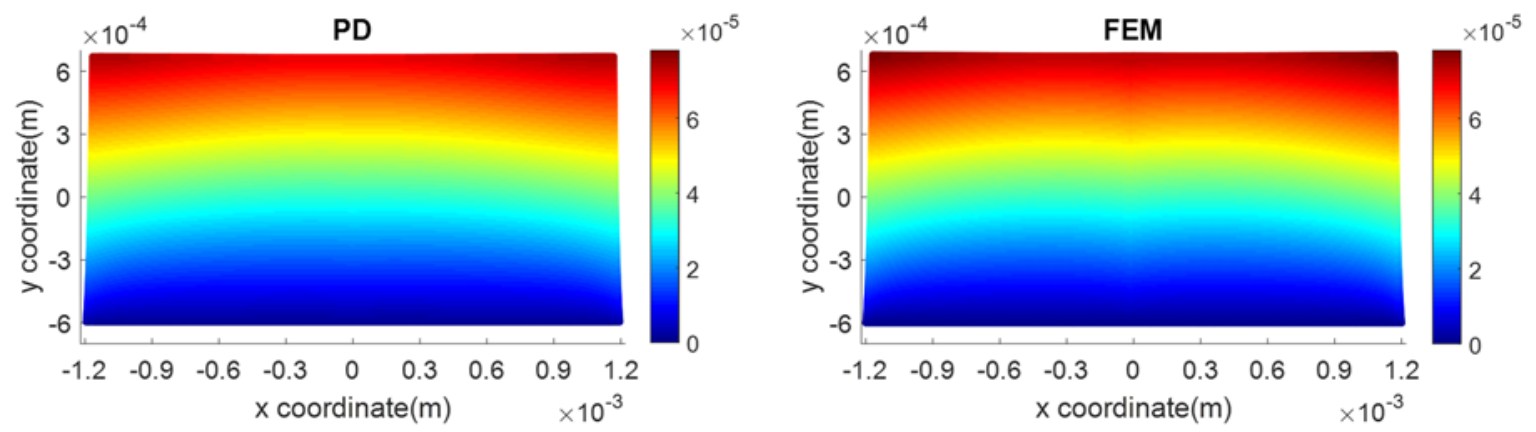

(b) Comparison of vertical displacement field for double grain model

Fig. 6 Comparison of displacement fields between PD and FEM for double grain model
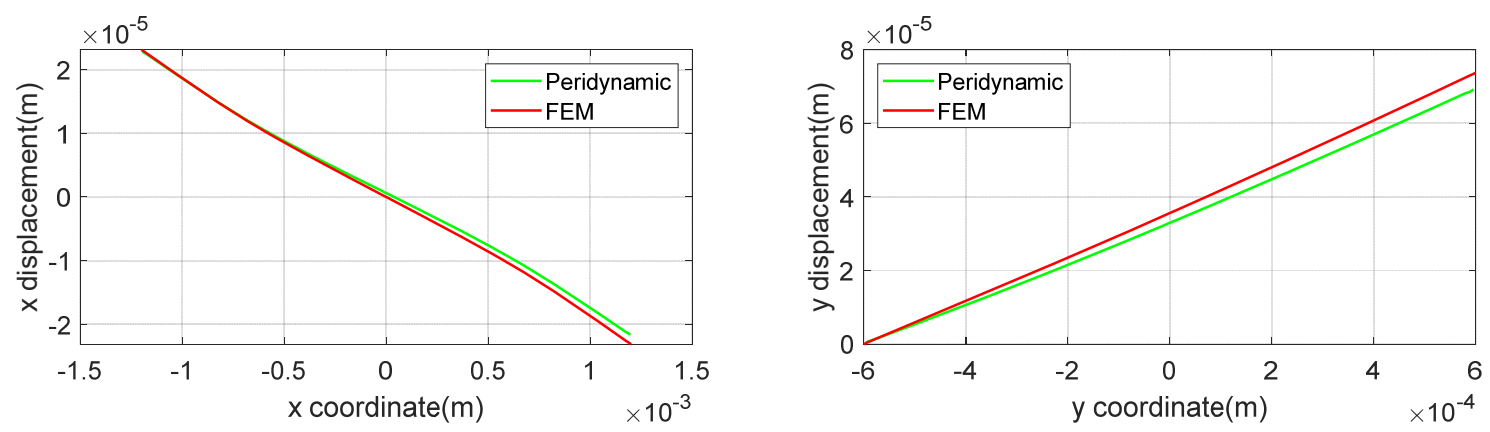

Fig. 7 Comparison of horizontal and vertical displacements along the central axes between PD and FEM for double grain model

As depicted in Figure 6 and 7, the results obtained from peridynamic and FEM show good agreement in horizontal and vertical displacements for double grain model. 


\subsection{Static Analysis of polycrystalline ice}

The polycrystalline ice modelled in this section consists of 100 randomly oriented grains (as shown in Figure 8) with the same ice properties used in the former examples. The plate has a length of $12 \mathrm{~mm}$ and a width of 12 $\mathrm{mm}$. The domain is discretized with uniform grids with 200 particles distributed along the horizontal and vertical directions. Similar to the previous simulations three layers of virtual particles are set along the bottom edge of the plate to constrain the bottom edge. The top edge is subjected to a vertical load of $P=600 \mathrm{MPa}$, applied as a body load through a volumetric region. The horizon radius is set as $\delta=1.809 \times 10^{-4} \mathrm{~m}$.

As shown in Figure 9 and 10, the numerical results based on the peridynamic theory match well with the results obtained from FEM. Only some small difference can be observed in the horizontal displacement field due to the approximate properties of the bonds between two different grains.

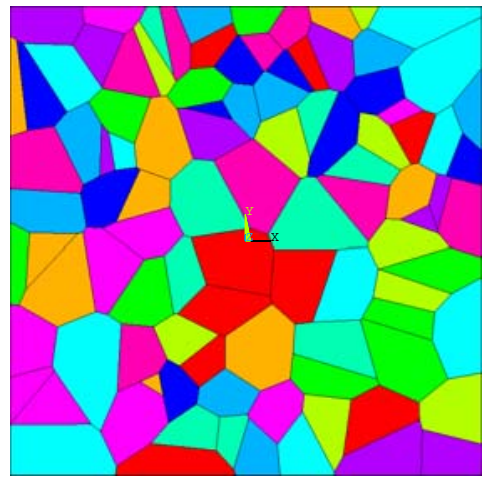

Fig. 8 The distribution of grains in the polycrystalline ice considered for static analysis
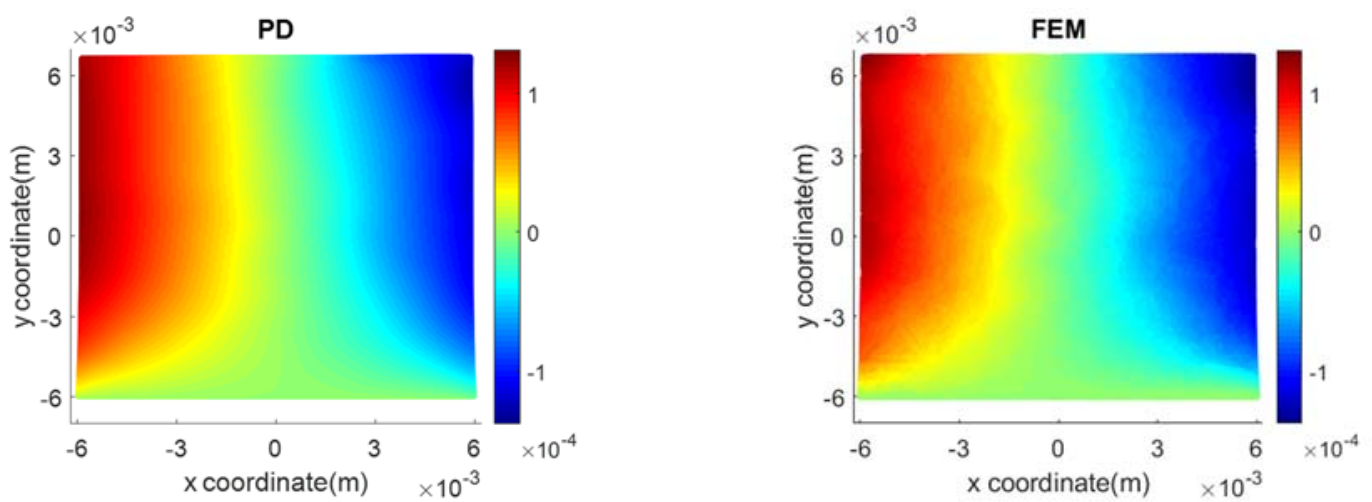

(a) Comparison of horizontal displacement field for polycrystalline ice
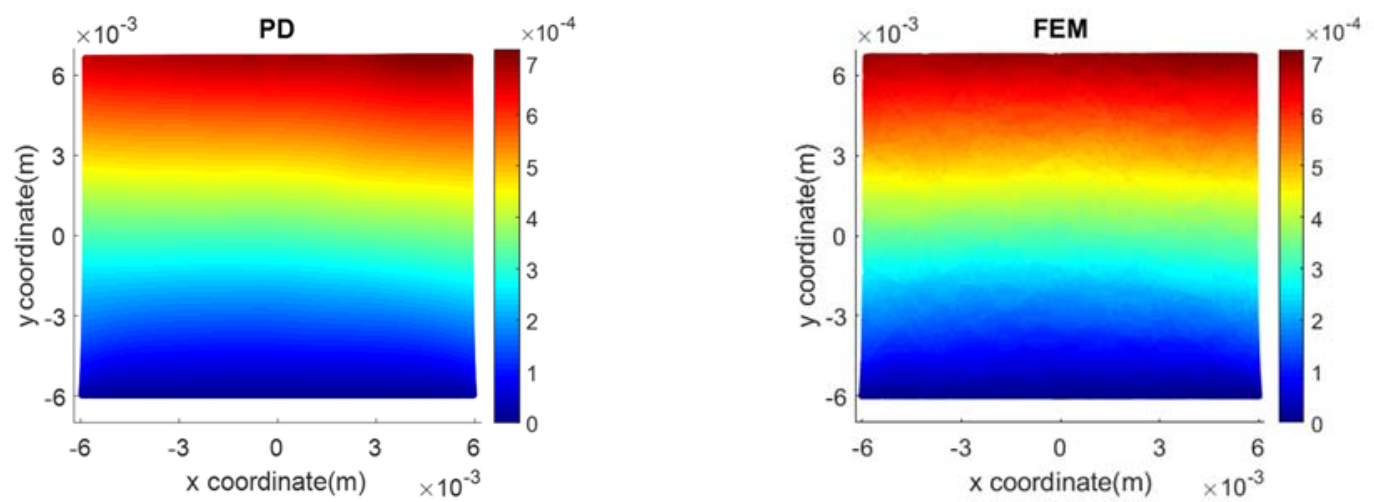

(b) Comparison of vertical displacement field for polycrystalline ice

Fig. 9 Comparison of displacement field between PD and FEM for polycrystalline ice 

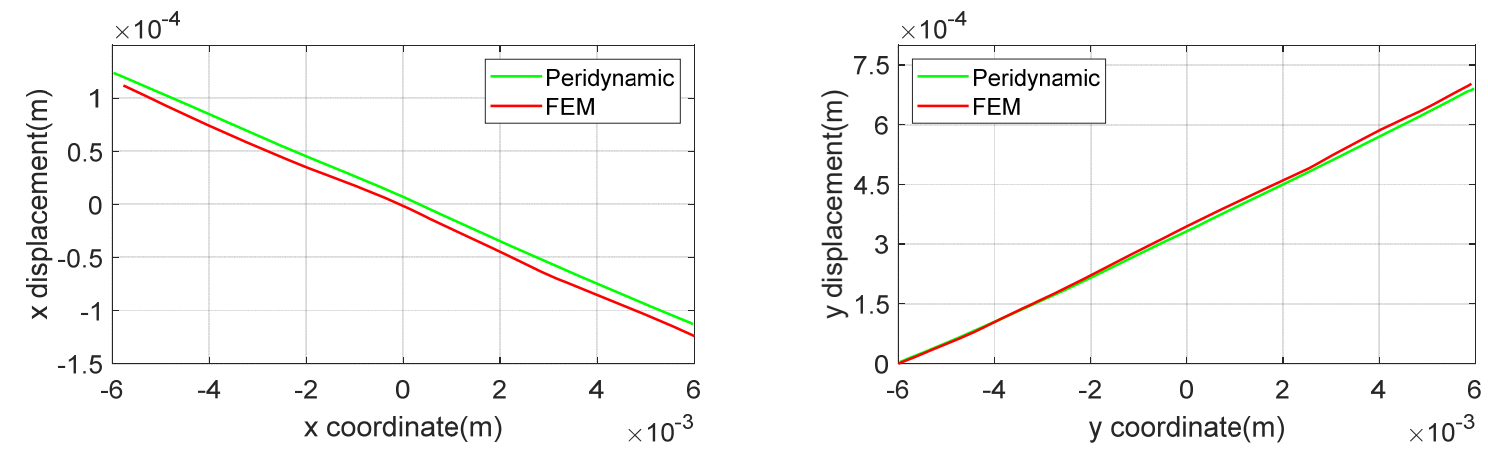

Fig. 10 Comparison of horizontal and vertical displacements along the central axis between PD and FEM for polycrystalline ice

\subsection{Dynamic Analysis of polycrystalline ice}

In this section, to investigate the fracture of polycrystalline ice, two pre-existing cracks are set at the top and the bottom edges of the plate with a length of $a_{0}=1.2 \mathrm{~mm}$. The ploycrystalline ice plate with a dimension of 15 $\mathrm{mm}$ in length and $15 \mathrm{~mm}$ in width is considered. 170 randomly oriented grains with average size of $1.15 \mathrm{~mm}$ are distributed inside the ice by using the Voronoi tessellation method, as depicted in Figure 11. According to Elvin [28], the average elastic modulus of the $15 \mathrm{~mm}$ by $15 \mathrm{~mm}$ sample is $9584 \mathrm{MPa}$, while the energy release rate of polycrystalline ice is considered as $G_{c}=2.6182 \mathrm{~Pa} \cdot \mathrm{m}$. To study the crack propagation, a horizontal velocity of $v_{0}=1.5 \mathrm{~m} / \mathrm{s}$ is added as a boundary condition along the left and right edges of the ice plate in a horizon region, as shown in Figure 12. Three virtual layers material points are set along both the left and right edges of the polycrystalline ice to apply the loading condition. The virtual layers of the material points are defined as no-fail zone due to the requirement of the appropriately transferring the external velocity load to the internal ice plate. To perform the time integration, an explicit central difference scheme is introduced with a time step size of $2.0 \times$ $10^{-9} \mathrm{~s}$. A total of 2500 time steps is utilised in the simulation for a total time of $5.0 \times 10^{-6} \mathrm{~s}$. In this study, three different $\mathrm{GBC}$ conditions $(0.5,1.0$ and 2.0$)$ and five different discretizations of ice model $(75 \times 75,100 \times$ $100,200 \times 200,300 \times 300$ and $400 \times 400$ paticles) are employed to analyse the propagation of the initial crack. Thus, the radius of horizon which is defined as $\delta=3.015 \Delta x$, varies with the discretisation size (corresponding to $0.603 \mathrm{~mm}, 0.45225 \mathrm{~mm}, 0.226125 \mathrm{~mm}, 0.15075 \mathrm{~mm}$ and $0.1130625 \mathrm{~mm}$ ).
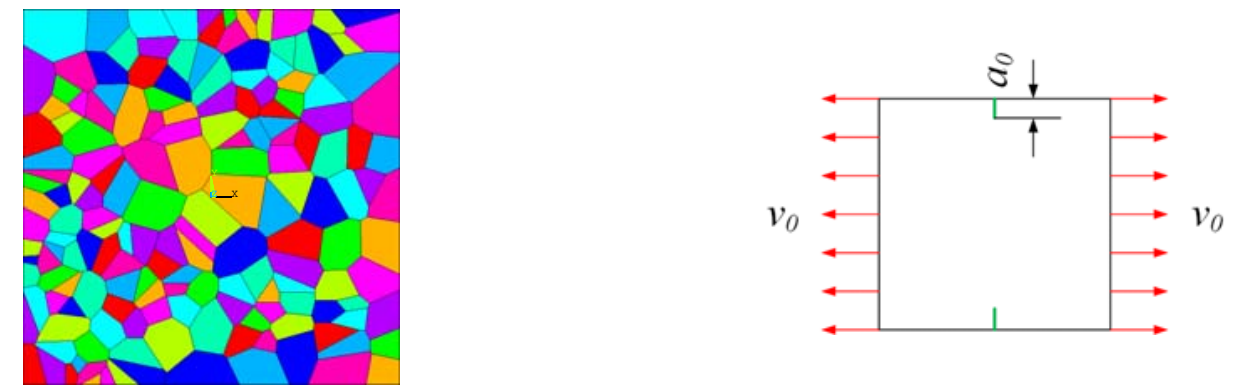

Fig. 11 The distribution of grains in the polycrystalline Fig. 12 The loading condition of the dynamic analysis ice (average size of $1.15 \mathrm{~mm}$ )

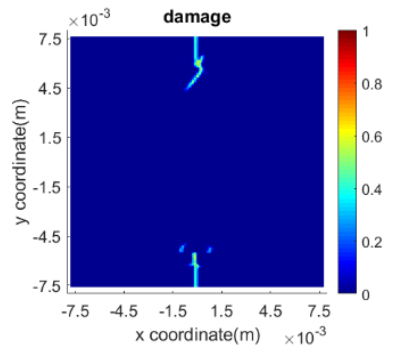

(a) $\mathrm{t}=2.6 \mu \mathrm{m}$

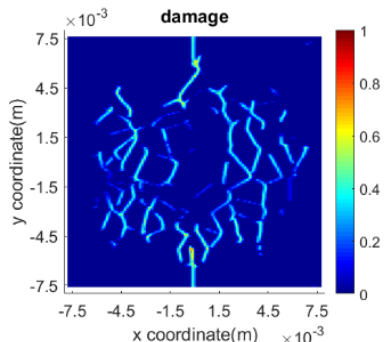

(b) $\mathrm{t}=3.2 \mu \mathrm{m}$

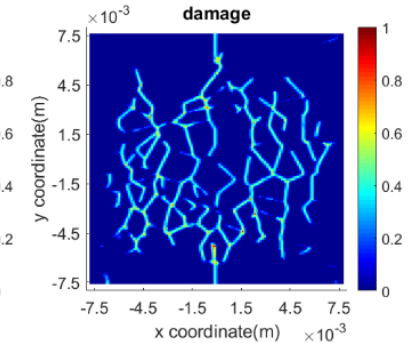

(c) $\mathrm{t}=3.5 \mu \mathrm{m}$

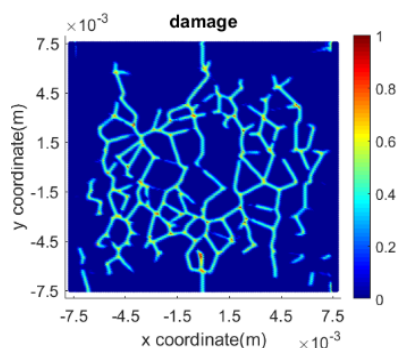

(d) $\mathrm{t}=4.0 \mu \mathrm{m}$

Fig. 13 The fracture of the polycrystalline ice with a horizon size of $0.226125 \mathrm{~mm}(200 \times 200$ particles $)$ $\mathrm{GBC}=0.5$ 
As shown in Figure 13, for the polycrystalline ice with an average grain size of $1.15 \mathrm{~mm}$ when the GBC is set as 0.5 initially, the pre-existing notch propagates and branches along the grain boundary. Then, some small fragmentations occur in the central part of the plate at grain boundaries due to the weakness of the bonds crossing grain boundaries. Under tensile loading, small cracks propagate and finally extend to the whole plate. The failure mode shows intergranular fracture pattern.

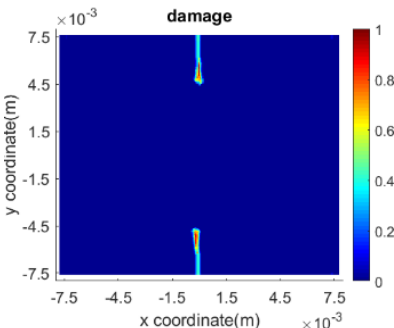

(a) $\mathrm{t}=3.2 \mu \mathrm{m}$

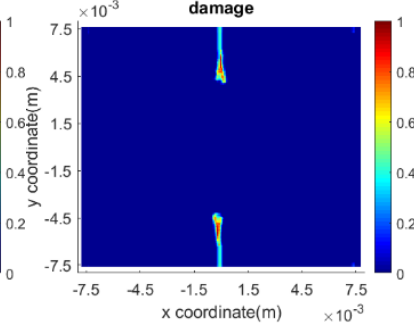

(b) $\mathrm{t}=3.5 \mu \mathrm{m}$

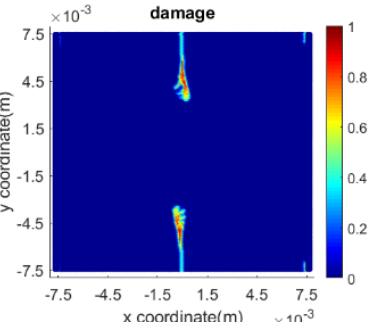

(c) $\mathrm{t}=4.0 \mu \mathrm{m}$

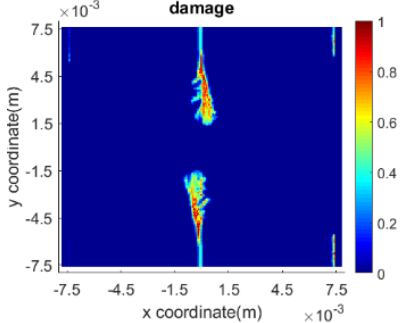

(d) $\mathrm{t}=5.0 \mu \mathrm{m}$

Fig. 14 The fracture of the polycrystalline ice with the horizon size of $0.226125 \mathrm{~mm}(200 \times 200$ particles $)$ $\mathrm{GBC}=1.0$

It is clearly seen in Figures 14 and 15 for $\mathrm{GBC}=1.0$ and $\mathrm{GBC}=2.0$, since the grain boundaries are not regarded as the weak parts of the polycrystalline ice, the propagation of the pre-existing crack is independent of the grain boundaries. The crack propagates through the grains and extend to the whole plate along the vertical direction. Thus, the failure mode is transgranular.

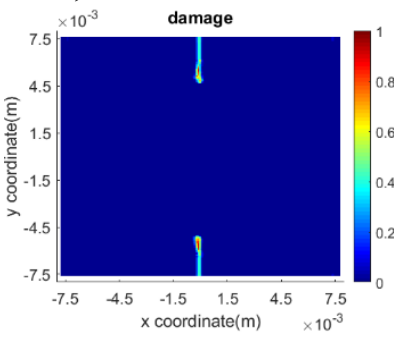

(a) $\mathrm{t}=3.2 \mu \mathrm{m}$

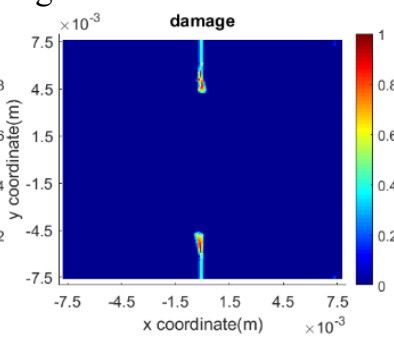

(b) $\mathrm{t}=3.5 \mu \mathrm{m}$

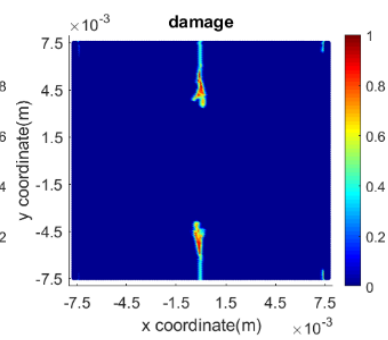

(c) $\mathrm{t}=4.0 \mu \mathrm{m}$

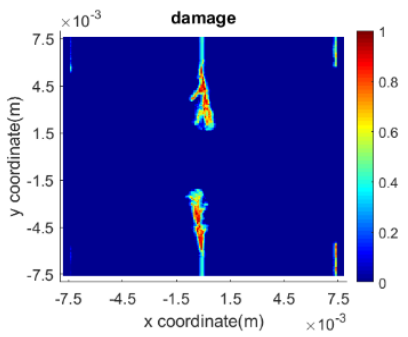

(d) $\mathrm{t}=5.0 \mu \mathrm{m}$

Fig. 15 The fracture of the polycrystalline ice with the horizon size of $0.226125 \mathrm{~mm}(200 \times 200$ particles $)$

$$
\mathrm{GBC}=2.0
$$

Figure 16 shows the comparison of the fracture of polycrystalline ice with different GBC and various discretization sizes. For $\mathrm{GBC}=0.5$, it can be seen clearly in the figure that the polycrystalline ice plate with larger horizon size and less material points inside, displays more serious damage, with more small cracks and fragmentations. For the horizon size equal to $0.603 \mathrm{~mm}, 0.45225 \mathrm{~mm}$, the major crack propagates and extends across the whole plate, while for the horizon radius of $0.1130625 \mathrm{~mm}$, only few small cracks occur in the ice plate, and the initial notch propagates and branches along the nearest grain boundary. However, with all different horizon sizes, the failure modes of the polycrystalline ice show intergranular and the failure patterns are similar. For $\mathrm{GBC}=1.0$ and $\mathrm{GBC}=2.0$, the ice plate with larger horizon size and fewer material points fails to capture the right propagation pattern of the major crack. Some small cracks occur at the boundary of the grains. Nevertheless, when the horizon radius is smaller than $0.226125 \mathrm{~mm}$, the fracture pattern shows convergent behavior, with major cracks only propagate through the grain. The results also show that the effect of the microstructure, i.e. grains, is important if the grain boundaries are weaker than the grain interiors whereas the effect of microstructure is insignificant for strong grain boundaries. 

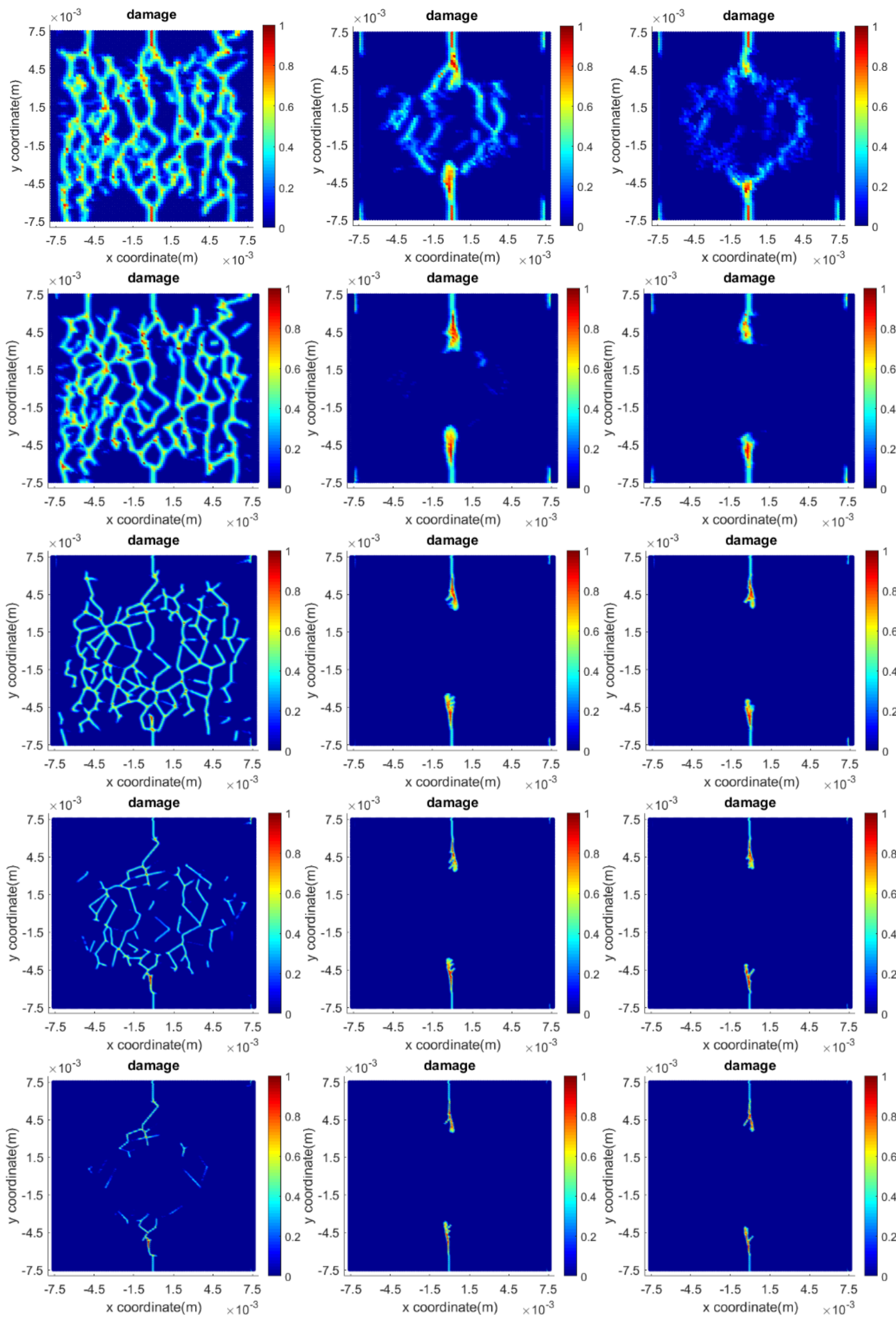

(a) $\mathrm{GBC}=0.5$

(b) $\mathrm{GBC}=1.0$

(c) $\mathrm{GBC}=2.0$

Fig. 16 The comparison of the fracture of polycrystalline ice with different GBC conditions (from left to right, valued as $0.5,1.0$ and 2.0) and different horizon size. (from top to the bottom, valued as $0.603 \mathrm{~mm}, 0.45225 \mathrm{~mm}, 0.226125 \mathrm{~mm}, 0.15075 \mathrm{~mm}$ and $0.1130625 \mathrm{~mm}$.) 


\section{CONCLUSION}

This paper presents a study of implementing the bond-based peridynamic theory, in which the equation of motion is an integral formulation rather than partial differential equation and is able to simulate the polycrystalline ice under uniaxial loading condition. In the simulations, freshwater columnar ice is considered as a transversely isotropic material. All grains in the ice are oriented randomly on the horizontal plane and modelled by using Voronoi tessellation method. The peridynamic parameters for the polycrystalline ice are calculated by equating the strain energy density of an individual material point based on classical continuum mechanics with peridynamics. The displacement fields along horizontal and vertical directions obtained from the numerical simulations are compared with the ones obtained from FEM showing good agreement between peridynamic and FEM results. Thus, the accuracy of the peridynamic polycrystalline ice model is successfully verified. In addition, the fracture of polycrystalline ice with different horizon sizes is also studied in this paper. With the $\mathrm{GBC}=0.5$, the failure mode is intergranular, which means that the initial crack and small fragmentations propagate and branch along the grain boundaries. On the other hand, for the $\mathrm{GBC}=1.0$ and $\mathrm{GBC}=2.0$, the major failure mode is transgranular. The initial notches propagate through the grain and extend across the whole plate. From these results, it can be concluded that the effect of the microstructure, i.e. grains, is important if the grain boundaries are weaker than the grain interiors whereas the effect of microstructure is insignificant for strong grain boundaries.

\section{ACKNOWLEDGEMENTS}

The authors gratefully acknowledges the financial support from China Scholarship Council (No. 201806680018) and University of Strathclyde.

\section{REFERENCES}

1. Gold, L.W., 1960. The cracking activity in ice during creep. Canadian Journal of Physics, 38(9), pp.11371148.

2. Gold, L.W., 1967. Time to formation of first cracks in ice. Physics of Snow and Ice. Proceedings of the International Conference on Low Temperature Science, pp.359-370.

3. Gold, L.W., 1999. Statistical characteristics for the strain-dependent density and the spatial position for deformation-induced cracks in columnar-grain ice. Journal of Glaciology, 45(150), pp.264-272.

4. Liu, F., Baker, I., Yao, G. and Dudley, M., 1992. Dislocations and grain boundaries in polycrystalline ice: a preliminary study by synchrotron X-ray topography. Journal of Materials Science, 27(10), pp.2719-2725.

5. Gay, P., Hirsch, P.B. and Kelly, A., 1954. X-ray studies of polycrystalline metals deformed by rolling. III. The physical interpretation of the experimental results. Acta Crystallographica, 7(1), pp.41-49.

6. Warner, D.H. and Molinari, J.F., 2006. Micromechanical finite element modeling of compressive fracture in confined alumina ceramic. Acta Materialia, 54(19), pp.5135-5145.

7. Sfantos, G.K. and Aliabadi, M.H., 2007. A boundary cohesive grain element formulation for modelling intergranular microfracture in polycrystalline brittle materials. International journal for Numerical Methods in Engineering, 69(8), pp.1590-1626.

8. Sukumar, N., Srolovitz, D.J., Baker, T.J. and Prévost, J.H., 2003. Brittle fracture in polycrystalline microstructures with the extended finite element method. International Journal for Numerical Methods in Engineering, 56(14), pp.2015-2037.

9. Paavilainen, J., Tuhkuri, J. and Polojärvi, A., 2006. Discrete element simulation of ice pile-up against an inclined structure. In IAHR 18th International Symposium on Ice. pp.177-184.

10. Paavilainen, J., Tuhkuri, J. and Polojärvi, A., 2011. 2D numerical simulations of ice rubble formation process against an inclined structure. Cold Regions Science and Technology, 68(1-2), pp.20-34.

11. Lu, W., Lubbad, R. and Løset, S., 2014. Simulating ice-sloping structure interactions with the cohesive element method. Journal of Offshore Mechanics and Arctic Engineering, 136(3), p.031501.

12. Feng, D., Dai Pang, S. and Zhang, J., 2016, June. Parameter Sensitivity in Numerical Modelling of IceStructure Interaction With Cohesive Element Method. In ASME 2016 35th International Conference on Ocean, Offshore and Arctic Engineering (pp. V008T07A012-V008T07A012). American Society of Mechanical Engineers.

13. Das, J., Polić, D., Ehlers, S. and Amdahl, J., 2014, June. Numerical simulation of an ice beam in four-point bending using SPH. In ASME 2014 33rd International Conference on Ocean, Offshore and Arctic Engineering (pp. V010T07A013-V010T07A013). American Society of Mechanical Engineers.

14. Gribanov, I., Taylor, R. and Sarracino, R., 2018, October. Application of cohesive zone model to the fracture 
process of freshwater polycrystalline ice under flexural loading. In IOP Conference Series: Earth and Environmental Science, 1(1), p. 012013.

15. Gribanov, I., Taylor, R. and Sarracino, R., 2018. Cohesive zone micromechanical model for compressive and tensile failure of polycrystalline ice. Engineering Fracture Mechanics, 196, pp.142-156.

16. Crocker, A.G., Flewitt, P.E.J. and Smith, G.E., 2005. Computational modelling of fracture in polycrystalline materials. International Materials Reviews, 50(2), pp.99-125.

17. Silling, S.A., 2000. Reformulation of elasticity theory for discontinuities and long-range forces. Journal of the Mechanics and Physics of Solids, 48(1), pp.175-209.

18. Askari, E., Bobaru, F., Lehoucq, R.B., Parks, M.L., Silling, S.A. and Weckner, O., 2008. Peridynamics for multiscale materials modeling. In Journal of Physics: Conference Series 125(1), p. 012078.

19. De Meo, D., Zhu, N. and Oterkus, E., 2016. Peridynamic modeling of granular fracture in polycrystalline materials. Journal of Engineering Materials and Technology, 138(4), p.041008.

20. Ghajari, M., Iannucci, L. and Curtis, P., 2014. A peridynamic material model for the analysis of dynamic crack propagation in orthotropic media. Computer Methods in Applied Mechanics and Engineering, 276, pp.431-452.

21. Silling, S.A. and Askari, E., 2005. A meshfree method based on the peridynamic model of solid mechanics. Computers \& Structures, 83(17-18), pp.1526-1535.

22. Gagliardini, O., Gillet-Chaulet, F. and Montagnat, M., 2009. A review of anisotropic polar ice models: from crystal to ice-sheet flow models. Physics of Ice Core Records, 2, pp.149-166.

23. Timco, G.W. and Weeks, W.F., 2010. A review of the engineering properties of sea ice. Cold Regions Science and Technology, 60(2), pp.107-129.

24. Oterkus, E. and Madenci, E., 2012. Peridynamic analysis of fiber-reinforced composite materials. Journal of Mechanics of Materials and Structures, 7(1), pp.45-84.

25. Oterkus, S., Madenci, E. and Agwai, A., 2014. Peridynamic thermal diffusion. Journal of Computational Physics, 265, pp.71-96.

26. Lebensohn, R.A., Montagnat, M., Mansuy, P., Duval, P., Meysonnier, J. and Philip, A., 2009. Modeling viscoplastic behavior and heterogeneous intracrystalline deformation of columnar ice polycrystals. Acta Materialia, 57(5), pp.1405-1415.

27. Hopkins, M.A., Frankenstein, S. and Thorndike, A.S., 2004. Formation of an aggregate scale in Arctic sea ice. Journal of Geophysical Research: Oceans, 109(C1).

28. Elvin, A.A., 1996. Number of grains required to homogenize elastic properties of polycrystalline ice. Mechanics of Materials, 22(1), pp.51-64.

29. Madenci, E. and Oterkus, E., 2014. Peridynamic theory and its applications. New York: Springer.

30. Oterkus, S. and Madenci, E., 2014. Fully coupled thermomechanical analysis of fiber reinforced composites using peridynamics. In 55th AIAA/ASMe/ASCE/AHS/SC Structures, Structural Dynamics, and Materials Co nference-SciTech Forum and Exposition 2014. 